\title{
Effects of end group functionalization and level alignment on electron transport in molecular devices
}

\author{
Gunn $\mathrm{Kim}^{\mathrm{a})}$ and Shuchun Wang \\ Center for High Performance Simulation and Department of Physics, North Carolina State University, \\ Raleigh, North Carolina 27695-7518, USA
}

\author{
Wenchang Lu, Marco Buongiorno Nardelli, and J. Bernholc ${ }^{\text {b) }}$ \\ Center for High Performance Simulation and Department of Physics, North Carolina State University, \\ Raleigh, North Carolina 27695-7518, USA and CSMD, Oak Ridge National Laboratory, Oak Ridge, \\ Tennessee 37881-6359, USA
}

(Received 1 October 2007; accepted 8 November 2007; published online 11 January 2008)

\begin{abstract}
The effect of metal-molecule coupling on electron transport is examined in the prototypical case of alkane chains sandwiched between gold contacts and bridged by either amine or thiol groups. The results show that end group functionalization plays a crucial role in controlling electron transport, and that the symmetries and spatial extent of orbitals near the Fermi level control the conductivity rather than the strength of the bonding. For amine/Au and thiol/Au junctions, a crossover in conductivity with increasing bias is predicted. () 2008 American Institute of Physics.
\end{abstract}

[DOI: $10.1063 / 1.2822180]$

\section{INTRODUCTION}

One of the main goals of nanotechnology is the miniaturization of electronic devices through exploitation of unique properties of matter at the nanoscale. This technological quest should lead to the development of novel applications and devices, where nanostructures, molecules, or quantum dots are the active elements of the device. At present, one of the most active areas of study is the development of molecular electronic devices. Advancements in such devices based on single organic molecules would enable new computer architectures and provide fresh insight into the nature of electronic coupling between inorganic solids and organic molecules. ${ }^{1,2}$ Although this field is relatively new, a large variety of techniques for measuring electron transport properties of single organic molecules have been developed. These include scanning tunneling microscopy, ${ }^{3,4}$ mechanical break junctions, ${ }^{5}$ and asymmetric metal/molecule/ nanoparticle/molecule/metal bridges. ${ }^{6,7}$ However, despite great efforts dedicated to the investigations of these systems, comprehensive conceptual understanding of electron transport in molecular systems is lacking, which impedes development of molecular devices and the technologies that would be based on them.

The primary aim of this work is to examine metal/ molecule coupling by directly comparing the conductance of long hydrocarbon (alkane) chains sandwiched between two gold contacts. Recent experiments revealed dramatic differences in electronic coupling at an alkane/gold interface depending on the end group: thiol $(-\mathrm{SH})$ versus amine $\left(-\mathrm{NH}_{2}\right) .^{7}$ In this letter, we examine the electron transport through these junctions through first-principles methods.

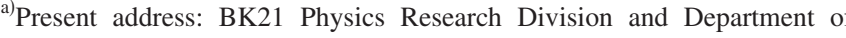
Physics Sungkyunkwan University, Suwon 440-746, Korea.

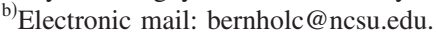

Analysis of the theoretical results shows that conductance is governed by the energies, spatial extent, and symmetries of the orbitals near the Fermi level rather than by the strength of the bonding. For the amine/Au and thiol/Au junctions, we find that in the low bias regime the current through the former is larger than through the latter, in agreement with recent experimental results. However, at biases higher than $\sim 1 \mathrm{~V}$, we predict that this ordering is reversed and a larger current flows through molecules with a thiol/Au contact. The reversal is explained through detailed analysis of individual orbitals and the shifts of their levels with bias. These shifts could be further tuned by gating or additional ligands, which could serve as a further avenue for adjusting the device performance.

\section{COMPUTATIONAL DETAILS}

Our calculations of the electronic structure and quantum transport are carried out using density functional theory, with generalized gradient approximation ${ }^{8}$ for evaluating the exchange-correlation energy. To obtain optimized geometries and electronic levels of our model structures, we employ the OPENMX code ${ }^{9-11}$ in a cluster geometry. The core electrons are described by Troullier-Martins-type pseudopotentials ${ }^{12}$ with a partial core correction ${ }^{13}$ in a separable form with multiple projectors. ${ }^{14}$ The atomic coordinates are relaxed until the Hellmann-Feynman forces are reduced to less than $0.03 \mathrm{eV} / \AA$.

After geometry optimization, quantum transport calculations are performed using a non-equilibrium Green's function method ${ }^{15,16}$ within a real-space multigrid-based approach. ${ }^{17,18}$ Ultrasoft pseudopotentials are employed to describe the core electrons. ${ }^{19}$ The various Green's functions and self-energies are computed in a basis of localized orbitals centered on atoms with a radius of $4.5 \AA$. The orbitals are variationally optimized for each system. ${ }^{20}$ Therefore, very 
few orbitals are needed for full density functional theory accuracy. We use four orbitals for hydrogen, six for carbon, eight for oxygen and nitrogen, and eleven for gold. The Hartree potential is obtained by solving the Poisson equation with fixed boundary conditions to match the semi-infinite gold leads on the left and right, respectively. To ensure that the bias-induced forces on the molecule are small, we consider only biases smaller than $1 \mathrm{eV}$.

The transmittance (transmission probability) spectra under the external applied bias $V$ are calculated using the wellknown formula, ${ }^{21}$

$$
T(E, V)=\operatorname{Tr}\left[\Gamma_{L}(E, V) G_{C}^{+}(E, V) \Gamma_{R}(E, V) G_{C}^{-}(E, V)\right],
$$

where $G_{C}^{ \pm}$are the advanced and retarded Green's functions of the conductor, while $\Gamma_{L, R}$ are the coupling functions of the conductor to the left and right leads. The current through the molecule is calculated from the Landauer-Büttiker formula $^{22,23}$

$$
I=\int_{-\infty}^{\infty} \frac{2 e^{2}}{h} T(E, V)\left[n_{\mathrm{FD}}\left(E-\mu_{R}\right)-n_{\mathrm{FD}}\left(E-\mu_{L}\right)\right] d E,
$$

where $\mu_{R(L)}$ is the chemical potential of the right (left) lead, $V=\left(\mu_{R}-\mu_{L}\right) / e$ and $n_{\mathrm{FD}}$ is the Fermi-Dirac distribution.

In our simulations, 1 molecule per $(4 \times 4)$ unit cell is adsorbed on a $\mathrm{Au}$ (111) surface and its structure is fully optimized. In analyzing the electronic structure and the molecular orbitals, the leads are represented by six bulklike layers of $\mathrm{Au}$ along the [111] direction. In transport calculations, the leads are semi-infinite and the scattering part includes the molecule and eight layers of $\mathrm{Au}$ on each side. Under bias, the charge density and potentials in the scattering part are computed self-consistently with open boundary conditions in the current direction and periodic boundary conditions in the other two directions. The charge density is allowed to change in the central region, which includes the molecules and two layers of $\mathrm{Au}$ on each side. It matches smoothly the bulk charge densities of the semi-infinite leads. While the calculations are carried out at $T=0$, the thermal broadening of the Fermi-Dirac function at the experimental (room) temperature is much smaller than level broadening induced by chemical coupling to the leads, for example.

\section{RESULTS AND DISCUSSION}

The optimized geometries of 1-pentanethiol (PT) $\left[\mathrm{CH}_{3}-\left(\mathrm{CH}_{2}\right)_{4}-\mathrm{SH}\right]$ and 1-pentylamine (PA) $\left[\mathrm{CH}_{3}-\left(\mathrm{CH}_{2}\right)_{4}-\mathrm{NH}_{2}\right]$ on $\mathrm{Au}$ (111) surfaces are shown in Figs. 1(a) and 1(b). For the PA molecule in Fig. 1(a), we find that the on-top adsorption site ${ }^{24,25}$ is the most favorable, with $\mathrm{Au}-\mathrm{N}$ distance of $2.35 \AA$. Considering the covalent bond radii of nitrogen $(\sim 0.75 \AA)$ and gold $(\sim 1.44 \AA)$, the value of $2.35 \AA$ is quite long. Furthermore, the uppermost Au layer is distorted due to the adsorption of the amino group. All the other sites, such as bridge, fcc-, and hcp-hollow result in a very weak or almost no binding. ${ }^{25}$ On the other hand, it is well known that the alkanethiol molecule prefers a fcchollow adsorption site. ${ }^{26-29}$ The optimized Au-S distance is $2.52 \AA$ and displays a covalent bond character, ${ }^{29}$ consistent with the covalent bond radius of sulfur, $\sim 1.02 \AA$. The ad- (a) PA

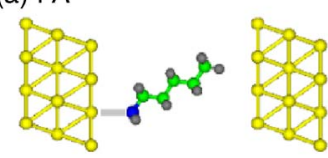

(c) ODA

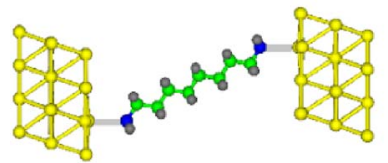

(b) PT

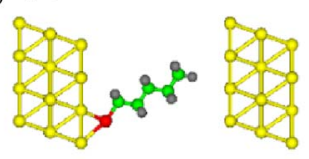

(d) ODT

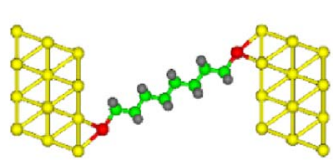

FIG. 1. (Color online) Atomic geometries for (a) 1-pentylamine (PA) and (b) 1-pentanethiol (PT) attached to gold surfaces and probed with an STM tip, modeled here by a gold surface. Panels (c) and (d) show the fully bridged surfaces of octanediamine (ODA) and octanedithiol (ODT), respectively. Yellow, green, gray, red, and blue balls represent gold, carbon, hydrogen, sulfur, and nitrogen atoms, respectively. See text.

sorption energy $[E($ molecule $)+E($ surface $)-E($ molecule + surface)] of the thiol group on gold is $2.3 \mathrm{eV}$, which is in agreement with the results of other groups. ${ }^{30,31}$ For the amino group on $\mathrm{Au}$ surface, we find an adsorption energy of $0.61 \mathrm{eV}$, which is a little higher than the adsorption energy of ammonia on $\mathrm{Au} .^{25}$ The relatively small adsorption energy and the distortion of the Au surface may explain why selfassembled monolayers of PA on gold are less stable than those of PT. ${ }^{7}$ According to our Mulliken population analysis, PT transfers a larger fraction of an electron $(0.65 e)$ to the gold surface than PA $(0.45 e)$. The structures of octanediamine (ODA) $\left[\mathrm{C}_{8} \mathrm{H}_{16}\left(\mathrm{NH}_{2}\right)_{2}\right]$ and octanedithiol (ODT) $\left[\mathrm{C}_{8} \mathrm{H}_{16}(\mathrm{SH})_{2}\right]$ on $\mathrm{Au}(111)$ surfaces are created, removing the methyl $\left(-\mathrm{CH}_{3}\right)$ terminal groups and using the same attachment geometries on the left as on the right [see Figs. 1(c) and $1(d)]$.

The PT and PA do not bond with the right lead and the distance between the molecule and the gold surface is set to $3.9 \AA$, which is the distance for physisorption of $\mathrm{CH}_{4}$ on $\mathrm{Au}$ surface. This distance could be even larger in experiment, depending on its conditions. Our previous calculations have shown that the shape of $I-V$ curve does not change much, while the amplitude of the current decreases exponentially with increasing distance. ${ }^{29}$

Self-consistent calculations for biases up to $1.0 \mathrm{~V}$ are performed for all four molecules sandwiched between the two gold leads. First, we discuss the cases of PT and PA. Figures 2(a)-2(c) show the transmittance spectra as a function of the incident electron energy under different biases, while the $I-V$ curves are displayed in Fig. 2(d). At zero bias, peak $\mathrm{A}$ at $-0.3 \mathrm{eV}$ is a signature of hybridized states between the molecular orbitals of PA and the left Au lead. Peak B at $-0.4 \mathrm{eV}$, which occurs in both PT and PA adsorption, stems from a surface state of the right Au lead. The appearance of these surface states has been previously documented in the literature. $^{32}$ Peak $\mathrm{C}$ at $-0.7 \mathrm{eV}$ originates from hybridized states between the molecular orbitals of PT and the left lead. In order to understand how the molecular orbitals correlate with the appearance of individual peaks in the transmittance and hence influence the current, we plot the highest occupied molecular orbital (HOMO) states in Fig. 3. As shown in Figs. 3(a) and 3(b), the HOMOs of both PA and PT are edge states 


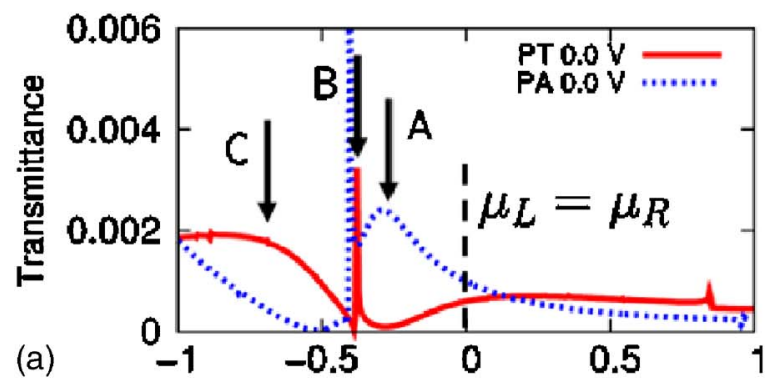

(a)
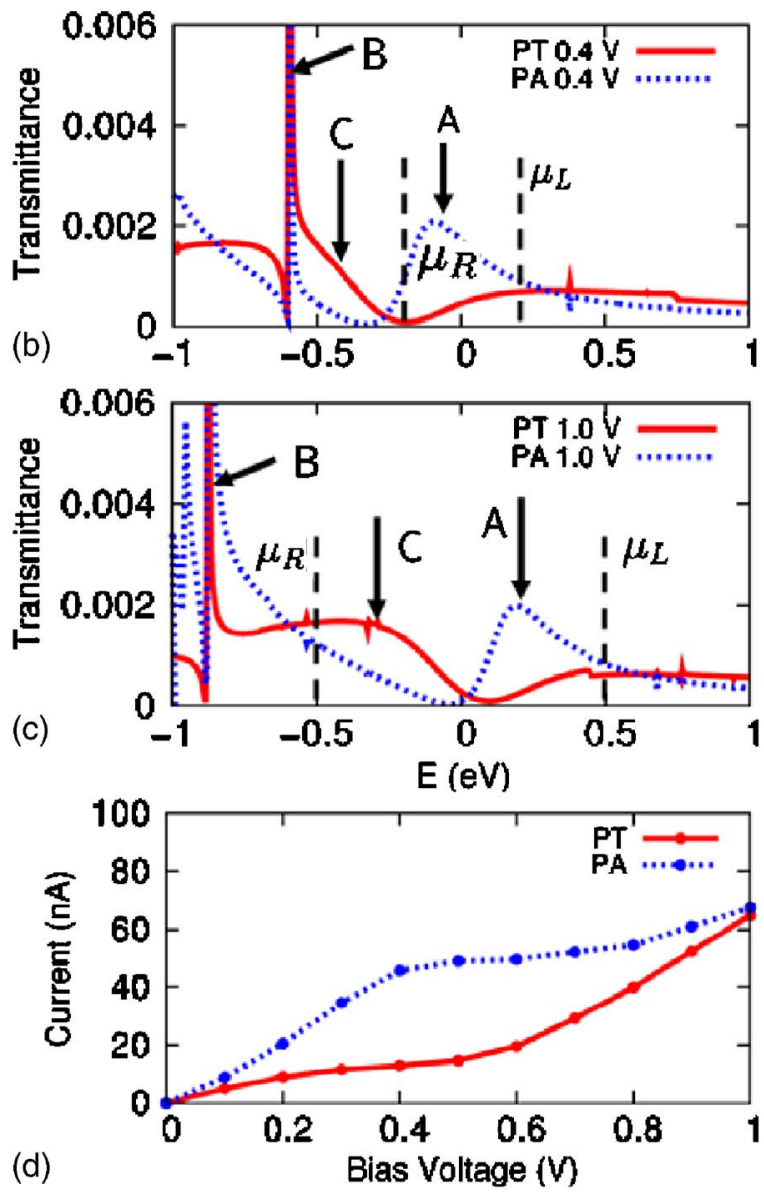

FIG. 2. (Color online) Transmittance spectra for PA (blue lines) and PT (red lines) as functions of the energy $E$ under biases of (a) $0.0 \mathrm{~V}$, (b) $0.4 \mathrm{~V}$, and (c) $1.0 \mathrm{~V}$. The zero of energy in taken in the middle of the bias window, between $\mu_{L}$ and $\mu_{R}$. (d) Current-voltage $(I-V)$ characteristics for PA (blue line) and PT (red line) on Au (111) surfaces, when probed by the model STM tip. See text.

localized on the amine and thiol groups, respectively, and located at approximately $0.3 \mathrm{eV}$ below the Fermi energy. The HOMO of PA derives from the lone pair of the nitrogen atom and is parallel to the axis of the alkane chain along which the current flows. This state results in the transmittance peak A. The HOMO of PT [Fig. 3(b)] is strongly localized at the edge, with a well-defined $p$-orbital character perpendicular to the current direction. Therefore, this state does not contribute to the conductance. In fact, the HOMO-1 derived state [Fig. $3(\mathrm{c})$ ], located at $0.7 \mathrm{eV}$ below the Fermi energy, is the transmitting state in the case of PT. It gives rise to the very broad peak $\mathrm{C}$ in the transmittance. Since broadening of the HOMO states is an indication of the strength of interaction between the molecule and the Au lead, we expect only a small broad- (a) HOMO of PA
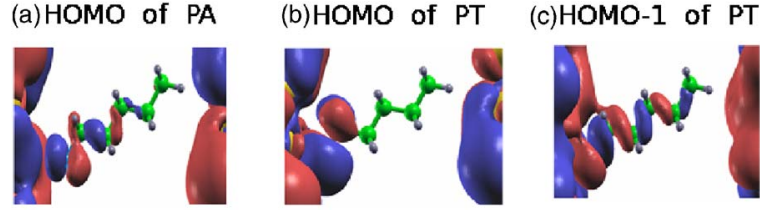

(d)HOMO of ODA
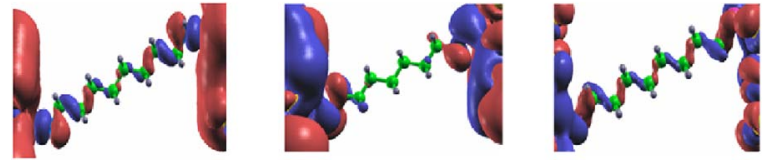

FIG. 3. (Color online) Wavefunctions of occupied orbitals of bridging molecules between two $\mathrm{Au}$ (111) surfaces at the $\Gamma k$ point: (a) HOMO of PA, (b) HOMO of PT, (c) HOMO-1 of PT, (d) HOMO of ODA, (e) HOMO of ODT, and (f) HOMO-1 of ODT. The values for the red and blue isodensity surfaces are $\pm 0.005 e / a_{0}^{3}$. See text.

ening for the amine end group, which is weakly bonded to the gold surface. In contrast, the thiol end group interacts with the gold surface via a strong chemical bond. ${ }^{33}$ Hence, substantial level broadening is anticipated. These expectations are consistent with the broadening observed in the transmittance spectra.

Figure 4 shows a schematic diagram of the alignment of the molecular orbitals that contribute to transmission probabilities of PA and PT relative to the chemical potentials of the two leads. Using the same notation as in Fig. 6, state A denotes the HOMO of PA, B is the surface state of the right lead and $\mathrm{C}$ is the HOMO-1 of the PT. The HOMO of PT is not shown in the figure because it does not contribute to the current. When bias is applied, the electrostatic potential drops mainly in the vacuum region and in the carbon chain. Since the surface state B is localized at the right lead, it is expected to be pinned by the chemical potential of this lead $\left(\mu_{R}\right)$. On the other hand, peaks $\mathrm{A}$ and $\mathrm{C}$, which are due to edge states at the end group (either amine for PA or thiol for PT), are expected to be pinned by the chemical potential of the left lead $\left(\mu_{L}\right)$. From Figs. 2(b) and 2(c), we can clearly see this effect. Peaks A and C shift up and peak B shifts down in energy with the increase of bias.

From 0.0 to $0.4 \mathrm{~V}$, tunneling through the $\mathrm{HOMO}$ of the PA (peak A) results in a nearly linear increase in the current [the blue line in Fig. 5(d)]. From $0.4 \mathrm{~V}$ on, the whole peak A falls into the bias window. Therefore, a plateau in the $I-V$ curve occurs. When the bias is larger than $0.8 \mathrm{eV}$, another

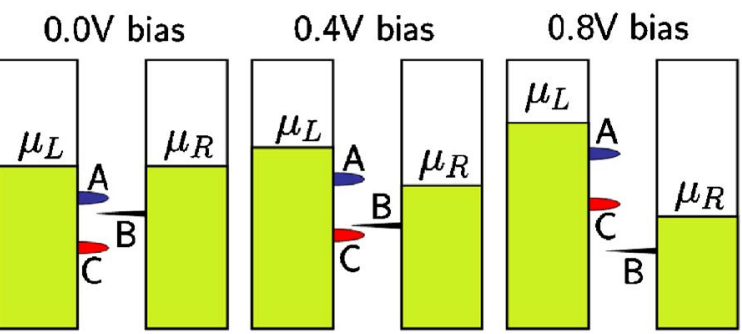

FIG. 4. (Color online) Schematic views of molecular levels of PA and PT and their alignment relative to the local chemical potentials under biases of $0.0,0.4$, and $0.8 \mathrm{~V}$. The state A is the HOMO of the PA, B is the surface state, and C is the HOMO- 1 of the PT. The HOMO of PT is not shown, since it is very localized and contributes little to conductance. 


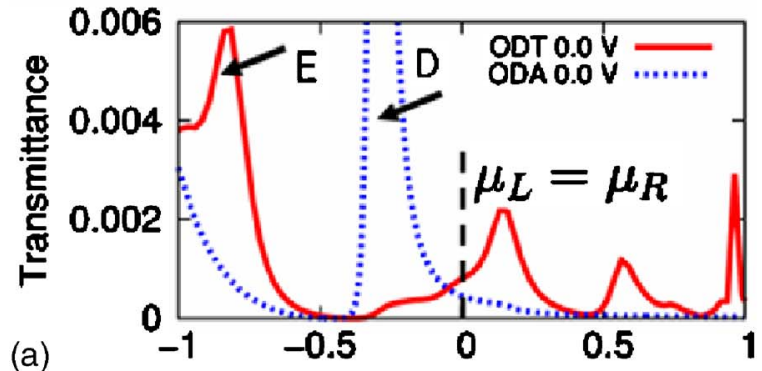

(a)
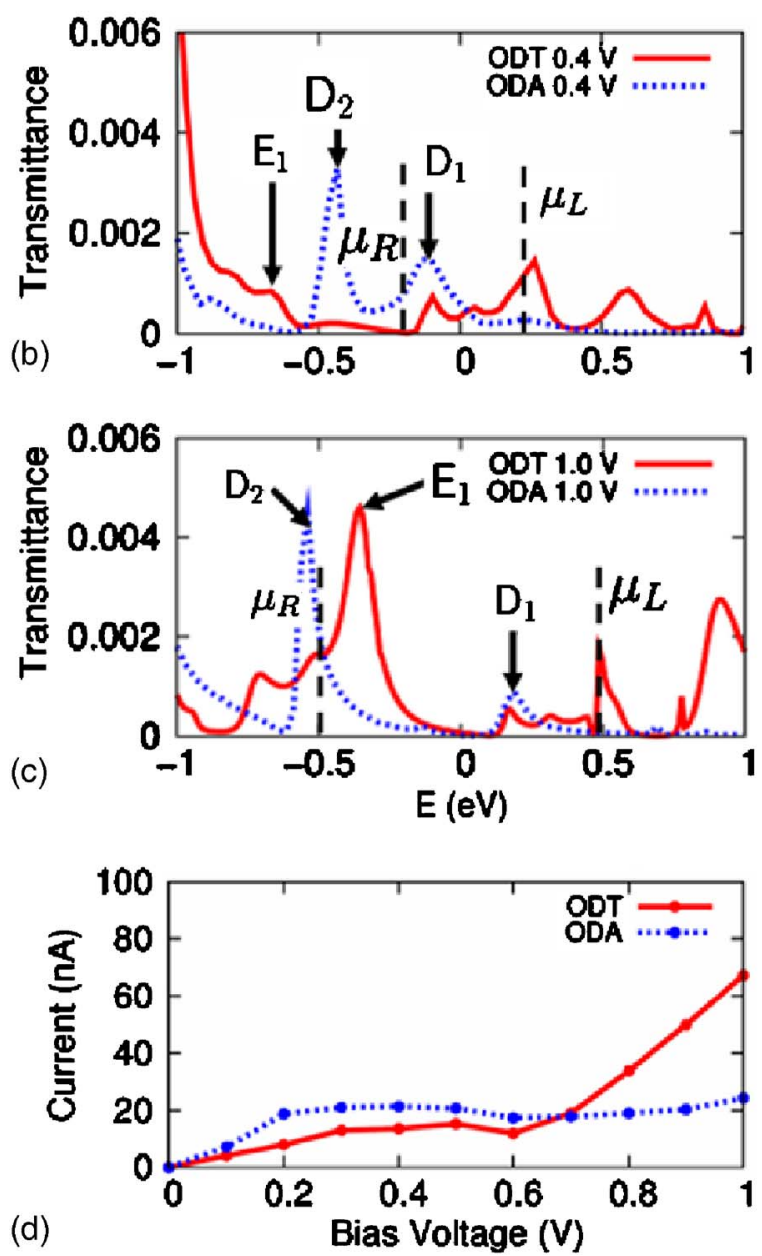

FIG. 5. (Color online) Transmittance spectra for ODA (blue lines) and ODT (red lines) as functions of the energy $E$ at biases of (a) $0.0 \mathrm{~V}$, (b) $0.4 \mathrm{~V}$, and (c) $1.0 \mathrm{~V}$. The zero of energy is taken in the middle of the bias window between $\mu_{L}$ and $\mu_{R}$. (d) Current-voltage (I-V) characteristics of ODA (blue line) and ODT (red line) bridging Au (111) surfaces.

state begins to contribute, leading to a slight increase in the current. In the case of PT, the current is very small for biases lesser than $0.6 \mathrm{~V}$. This is because the HOMO of PT is very localized and does not contribute much to the current, while the scattering state HOMO-1 is still far from the bias window. When the bias is larger than $0.6 \mathrm{~V}$, tunneling through HOMO-1 results in a linear increase in current.

Next, we turn to transport properties of ODA and ODT sandwiched between two Au leads. Both sides of the molecules are connected to the Au surface with either amine (ODA) or thiol (ODT) end groups, as shown in Figs. 5(c) and 5(d). Figure 5 shows the transmittance and $I-V$ curves for ODA (blue lines) and ODT (red lines). Obviously, there

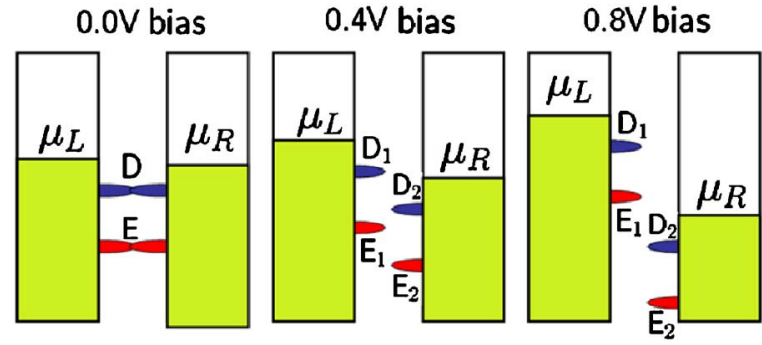

FIG. 6. (Color online) Schematic view of molecular levels of ODA and ODT and their alignment relative to the local chemical potentials at biases of $0.0,0.4$, and $0.8 \mathrm{~V}$. D is the HOMO of ODA and E is the HOMO- 1 of ODT. At finite biases, both $\mathrm{D}$ and $\mathrm{E}$ split, due to breakage of the inversion symmetry. See text.

is no surface-state-related peak for either ODT or ODA, since these molecules bond to both surfaces. In analogy to PA and PT, peak D in Fig. 5 is associated with the HOMO of ODA [Fig. 3(d)] and peak E is related to the HOMO-1 of ODT [Fig. 3(f)]. The HOMO of ODT (Fig. 3(e)) is very localized and practically does not contribute to the current. At zero bias, both systems have inversion symmetry and the HOMOs are nearly doubly degenerate. Under finite bias, the inversion symmetry is broken and the HOMOs are split into two states. Correspondingly, peak $\mathrm{D}$ splits into $\mathrm{D}_{1}$ and $\mathrm{D}_{2}$ when bias is applied. Peak $\mathrm{D}_{1}$ is pinned by $\mu_{L}$ and peak $\mathrm{D}_{2}$ is pinned by $\mu_{R}$. Similarly, peak $\mathrm{E}$ also splits into two ( $\mathrm{E}_{1}$ and $E_{2}$ ), but only $E_{1}$ is visible in Fig. 5, since $E_{2}$ is below $-1.0 \mathrm{eV}$. Depending on the bias voltage, the height of $\mathrm{E}_{1}$ varies dramatically. For example, large suppression of the conductance occurs at the bias of $0.4 \mathrm{~V}$, as seen in Fig. 5(b).

The same mechanisms as for PT and PA can be used to explain the different $I-V$ characteristics of ODA and ODT. At small bias, tunneling through $\mathrm{D}_{1}$ of ODA results in a larger current than in the ODT. At large biases, the HOMO-1 of ODT contributes strongly to the current, which results in ODT transmitting a larger current than ODA at biases larger than $0.7 \mathrm{~V}$. Figure 6 shows schematically the alignment of the molecular orbitals that contribute to the transmission probabilities of ODA and ODT at different voltages. The symbols used are the same as in Fig. 4.

In general, we find that electron tunneling through a molecular junction is strongly affected by coupling between the discrete energy levels in the molecule and the continuum states of the leads. Coupling with the continuum broadens the molecular levels, which become resonances. The shapes and symmetries of the transmitting wave functions result in different broadening of the various conductance peaks. In the adsorption of an amine on gold, which results in hybridization of the nitrogen lone pair with the gold surface, a hybridized state is directed along the bond axis, leading to a narrow transmittance, whereas more localized and covalent bonding at the junction, as in thiol/Au, results in a much broader transmittance. Furthermore, when the molecular orbitals are more delocalized, they overlap better with the lead states, ensuing in higher conductance. As the bias increases, the HOMO shifts into the bias window and thus the current increases. In the amine- and thiol-bridged alkanes, the current at low biases flows mainly through the HOMO. Until the HOMO-1 enters the bias window, the $I-V$ curve has a pla- 
teau. Among our model systems, the ODA has the largest energy spacing between the HOMO and HOMO-1 levels, and thus the longest plateau in the $I-V$ curve. While our results for current flow through $\mathrm{PT}$ and PA reproduce the low-bias experimental trends, they predict that transmission through PT will eventually become larger for voltages greater than $1 \mathrm{eV}$ in our configuration. For fully bridged leads, the current through ODT becomes greater than through ODA at about $0.7 \mathrm{eV}$ in our geometry.

\section{CONCLUSION}

In summary, the electronic structure and transport properties of long alkane chains sandwiched between gold contacts was examined theoretically, focusing on metal/molecule coupling and its effect on the electronic transport. We found that end group functionalization plays a crucial role in controlling the electronic transport. At small biases $(<0.6 \mathrm{~V})$, the amine end group results in a larger current than the thiol end group, although the former is only weakly bonded to the surface, while thiols are well known to chemisorb strongly at gold surfaces. It follows that the $I-V$ characteristics are mainly determined by the properties (symmetry and decay length) of the molecular orbitals near the Fermi level, and by the number of orbitals falling within the bias window and thus contributing to the current rather than by the strength of the bond. Our results are in good agreement with the available experimental data for similar systems. ${ }^{7}$

\section{ACKNOWLEDGMENTS}

We would like to thank Professor G. N. Parsons for sharing his data prior to publication and for helpful discussions. We gratefully acknowledge support by DOE and ONR, and grants of supercomputer time provided by the DOD Challenge Program and the DOE INCITE Program.

${ }^{1}$ Y. Selzer, A. Salomon, and D. J. Cahen, J. Phys. Chem. B 106, 10432 (2002).

${ }^{2}$ A. Nitzan and M. A. Ratner, Science 300, 1384 (2003).

${ }^{3}$ X. D. Cui, X. Zarate, J. Tomfohr, O. F. Sankey, A. Primak, A. L. Moore, T. A. Moore, D. Gust, G. Harris, and S. M. Lindsay, Nanotechnology 13, 5 (2002).
${ }^{4}$ C. Joachim, J. K. Gimzewski, R. R. Schlittler, and C. Chavy, Phys. Rev. Lett. 74, 2102 (1995).

${ }^{5}$ C. Kergueris, J. P. Bourgoin, S. Palacin, D. Esteve, C. Urbina, M. Magoga, and C. Joachim, Phys. Rev. B 59, 12505 (1999).

${ }^{6}$ I. Amlani, A. M. Rawlett, L. A. Nagahara, and R. K. Tsui, Appl. Phys. Lett. 80, 2761 (2002).

${ }^{7}$ C. Chu, J.-S. Na, and G. N. Parsons, J. Am. Chem. Soc. 129, 2287 (2007).

${ }^{8}$ J. P. Perdew, K. Burke, and M. Ernzerhof, Phys. Rev. Lett. 77, 3865 (1996).

${ }^{9}$ T. Ozaki, Phys. Rev. B 67, 155108 (2003).

${ }^{10}$ T. Ozaki and H. Kino, Phys. Rev. B 69, 195113 (2004).

${ }^{11}$ The density functional theory code, OpenMX, the basis orbitals, and the pseudopotentials used in this study are available on the web site http://www.openmx-square.org under the conditions of the GNU General Public License.

${ }^{12}$ N. Troullier and J. L. Martins, Phys. Rev. B 43, 1993 (1991).

${ }^{13}$ S. G. Louie, S. Froyen, and M. L. Cohen, Phys. Rev. B 26, 1738 (1982).

${ }^{14}$ P. E. Blochl, Phys. Rev. B 41, 5414 (1990).

${ }^{15}$ B. Larade, J. Taylor, H. Mehrez, and H. Guo, Phys. Rev. B 64, 075420 (2001).

${ }^{16}$ M. Brandbyge, J.-L. Mozos, P. Ordejón, J. Taylor, K. Stokbro, Phys. Rev. B 65, 165401 (2002)

${ }^{17}$ M. Buongiorno Nardelli, J.-L. Fattebert, and J. Bernholc, Phys. Rev. B 64, 245423 (2001).

${ }^{18}$ W. Lu, V. Meunier, and J. Bernholc, Phys. Rev. Lett. 95, 206805 (2005).

${ }^{19}$ D. Vanderbilt, Phys. Rev. B 41, 7892 (1990).

${ }^{20}$ J.-L. Fattebert and J. Bernholc, Phys. Rev. B 62, 1713 (2000).

${ }^{21}$ S. Datta, Electronic Transport in Mesoscopic Systems (Cambridge University Press, Cambridge, 1995).

${ }^{22}$ R. Landauer, Philos. Mag. 21, 863 (1970).

${ }^{23}$ M. Büttiker, Phys. Rev. Lett. 57, 1761 (1986).

${ }^{24}$ L. Venkataraman, J. E. Klare, I. W. Tam, C. Nuckolls, M. S. Hybertsen, and M. L. Steigerwald, Nano Lett. 6, 458 (2006).

${ }^{25}$ A. Bilić, J. R. Reimers, N. S. Hush, and J. Hafner, J. Chem. Phys. 116, 8981 (2002)

${ }^{26}$ F. T. Arce, M. E. Vela, R. C. Salvarenzza, and A. J. Aevia, Langmuir 14, 7203 (1998).

${ }^{27}$ Y. Yourdshahyan, H. K. Zhang, and A. M. Rappe, Phys. Rev. B 63, 081405 (2001).

${ }^{28}$ H. Gronbeck, A. Curioni, and W. Andreoni, J. Am. Chem. Soc. 122, 3839 (2000).

${ }^{29}$ S. Wang, W. Lu, Q. Zhao, and J. Bernholc, Phys. Rev. B 74, 195430 (2006).

${ }^{30}$ C. Majumder, H. Mizuseki, and Y. Kawazoe, J. Chem. Phys. 118, 9809 (2003)

${ }^{31}$ Y. Yourdshahyan and A. M. Rappe, J. Chem. Phys. 117, 825 (2002).

${ }^{32}$ S. LaShell, B. A. McDougall, and E. Jensen, Phys. Rev. Lett. 77, 3419 (1996)

${ }^{33}$ W. Tian, S. Datta, S. Hong, R. Reifenberger, J. T. Henderson, and C. P. Kubiak, J. Chem. Phys. 109, 2874 (1998). 